\title{
乌药烷型倍半萜及其二聚体的全合成研究进展
}

\author{
乐贵洲 ${ }^{a, c}$ 杨 立 $^{a}$ 袁长春 $^{a} \quad$ 杜 虎 ${ }^{a} \quad$ 刘 波*,a,b \\ $\left({ }^{a}\right.$ 四川大学化学学院 绿色化学与技术教育部重点实验室 成都 610064) \\ ${ }^{b}$ 中国科学院上海有机化学研究所 中国科学院天然产物合成化学重点实验室 上海 200032) \\ $\left({ }^{c}\right.$ 四川农业大学生命科学与理学院 雅安 625014)
}

\begin{abstract}
摘要 乌药烷型倍半萜及其二聚体是一大类具有 cis,trans-3/5/6三并环特殊骨架的天然产物, 主要是从金粟兰科植物中 分离得到. 大多数乌药烷型倍半萜及其二聚体具有显著的生物活性, 如抗真菌、对 B 细胞有毒性介导的免疫抑制及对 延迟整流钾电流有选择性的抑制作用等. 综述了国内外有机化学家对该类天然产物的全合成研究进展.

关键词 乌药烷; 倍半萜; 二聚体; 全合成
\end{abstract}

\section{Progress in Total Syntheses of Lindenane-Type Sesquiterpenoids and Their Dimers}

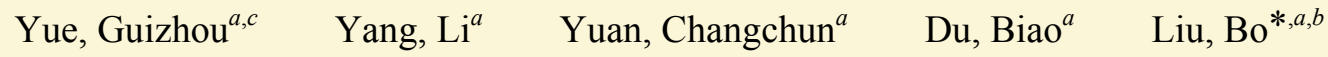 \\ ( ${ }^{a}$ Key Laboratory of Green Chemistry \& Technology of Ministry of Education, College of Chemistry, Sichuan University, \\ Chengdu 610064) \\ ( ${ }^{b}$ Key Laboratory of Synthetic Chemistry of Natural Substances, Shanghai Institute of Organic Chemistry, \\ Chinese Academy of Sciences, Shanghai 200032) \\ ( ${ }^{c}$ School of Life and Science, Sichuan Agricultural University, Ya'an 625014)
}

\begin{abstract}
Lindenane-type sesquteripenoids and their dimers, mainly isolated from chloranthaceae, are a big family of natural products, which possess novel polycyclic framework embedded with a sterically congested cyclopentane (ring B), an unusual trans-5/6 ring junction, and an angular methyl group. Moreover, many of them exhibited impressing bioactivities, for example remarkable antifungal activity, toxicity-mediated immunosuppression against B cells, and selective inhibition on the delayed rectifier $\mathrm{K}^{+}$current. In this review, the progress in the total syntheses of lindenane-type sesquiterpenoids and their dimmers is summarized.
\end{abstract}

Keywords lindenane; sesquiterpenoid; dimer; total synthesis

金粟兰科(Chloranthaceae) 是只有四大类的小科植 物(Sarcandra, Chloranthus, Hedyosmum 及 Ascarina), 大 约有 70 种该类植物主要分布在热带及亚热带地区, 其 中三大类及 16 种植物在中国南部有分布. 我国的金粟 兰属(Chloranthus swartz)植物大多以全草入药，在民间 已有广泛应用，具有社风散寒、舒精强骨、活血散瘀及 消肿止痛等功效 ${ }^{[1]}$. 长久以来, 天然产物化学家从金粟 兰科药用植物中分离得到多个系列具有特殊结构和良 好生物活性的分子, 引起了药物及有机化学家们的广泛 关注. 其中萜类化合物, 尤其是倍半萜内酯类化合物数
量众多，而且具有抗菌、抗病毒、抗溃疡、镇痛、抗肿 瘤、抗血小板聚集等生物活性. 在倍半萜类中又以乌药 烷型(又称钓樟烷型)倍半萜(lindenane-type sesquiterpenoid)及其二聚体居多，这类天然产物被认为是金粟兰 科植物化学分类学标志性的化合物 ${ }^{[2]}$, 最近有多篇综述 报道了该类天然产物 ${ }^{[2,3]}$. 它们的提取最早始于 1925 年 乌药烯(lindene 1) 的报道 ${ }^{[4]}$ (Chart 1), 随后又陆续报道了 乌药烯 $\mathbf{2}^{[5]}$, 金粟兰内酯(chlorathlactones $\mathrm{A} \sim \mathrm{G}$ ) ${ }^{[6]}$, 银线 草内酯(shizukanolides $\mathrm{A} \sim \mathrm{F})^{[7]}$, 9-hydroxy heterogorgiolide $^{[8]}$, onoseriolide ${ }^{[9]}$, oxyonoseriolide ${ }^{[10]}$, chloranjapolides

*E-mail: chembliu@scu.edu.cn

Received July 3, 2012; revised August 31, 2012; published online September 5, 2012.

Project supported by the National Natural Science Foundation of China (Nos. 20872098, 21021001, 21172154), the National Basic Research Program of China (973 Program, No. 2010CB833200).

国家自然科学基金(Nos. 20872098, 21021001, 21172154)、国家重点基础研究发展规划(973 计划, No. 2010CB833200). 
$\mathrm{A} \sim \mathrm{E}^{[11]}$, strychinstenolide 6-O-acetates $\mathrm{A}, \mathrm{B}^{[12]}$ 等 $^{[13]}$. 它 们的二聚体也被大量的分离鉴定出来(Chart 2), 如: chlorahololides $\mathrm{A} \sim \mathrm{F}^{[14]}$, 银线草醇 $(\text { shizukaols } \mathrm{A} \sim \mathrm{J})^{[15]}$, cycloshizukaol $\mathrm{A}^{[16]}$, chloramultilides $\mathrm{A} \sim \mathrm{D}^{[17]}$, tianmushanol 和 8-O-methyltianmushanol ${ }^{[18]}$, 穗金粟兰醇 (chloramultiols $\mathrm{A} \sim \mathrm{F})^{[19]}$, 含有过氧键的 spicachlorantins $\mathrm{A} \sim \mathrm{F}^{[20]}$, sarcandrolides $\mathrm{A} \sim \mathrm{E}^{[21]}$, henriols $\mathrm{A} \sim \mathrm{D}^{[22]}$, chlorajaponilides $\mathrm{A} \sim \mathrm{E}^{[23]}$, sarcanolides $\mathrm{A}, \mathrm{B}^{[24]}$ 等. 三聚 体 trishizukaol ${ }^{[15 \mathrm{~d}]}$ 及更复杂的多环天然产物 bolivianine 及 isobolivianine ${ }^{[25]}$ 在最近也被报道. 目前该类化合物有 近 100 种, 并且新的结构类似物不断被分离鉴定出来, 其中许多化合物显示了良好的生物活性, 如：抗真

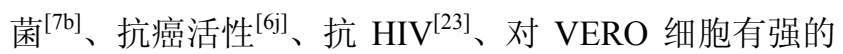

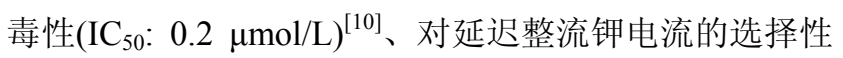

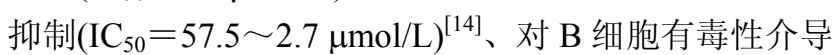
的免疫抑制 ${ }^{[26]}$ 、对细胞黏着分子的表达具有很强的抑制 作用 (有效浓度达到 $\mathrm{nmol} / \mathrm{L}$ 级 $)^{[27]}$ 以及对 II 型糖尿病、 肥胖症及其引起的并发症显示疗效 ${ }^{[28]}$ 等.

从结构上看, 乌药烷型倍半萜及其二聚体都具有相 同的核心骨架, 主要的结构特点如下：(1)一个手性季碳 C-10; (2)一个张力较大的 trans-5/6 并环 BC 体系; (3) 一 个[3.1.0]己烷 $\mathrm{AB}$ 体系, 14 位的甲基与环丙烷处于 $\mathrm{B}$ 环 的同侧, 使得 $\mathrm{B}$ 环相当拥挤; (4)除了以上特点外, 在大
多数二聚体中还有一个连接两个片段、位阻较大的六元 环. 曾有人提出大多数二聚体的生源假说：双烯片段 I 和亲双烯片段 II 进行分子内极性反转的 endo-DielsAlder 反应构建六元环, 再转化为各种不同的二聚 体 $^{[14 a, 15 a, 24]}$ (Scheme 1). 以上的结构特点使得乌药烷型倍 半萜及其二聚体的合成都具有较大的挑战性, 引起了国 内外有机化学家的关注. 尤其是最近几年, 我国有机化 学家对该类化合物进行了较多的全合成研究. 本文按照 论文发表的时间顺序加以综述及评论.

\section{1 乌药烷型倍半萜及其二聚体的全合成研究}

\subsection{Baldwin 小组}

2007 年牛津大学的 Baldwin 小组 ${ }^{[29]}$ 报道了 lindene 2 的全合成研究，从商品化原料 Hagemann's ester 出发, 利用 $\mathrm{Cu}(\mathrm{II})$ 的卡宾物种对烯烃的插入反应构建 $3 / 5$ 并环 为关键反应 (Scheme 2). 合成路线如下: 在 $\mathrm{CuBr} \bullet \mathrm{SMe}_{2}$ 催化下，乙烯基溴化镁对 Hagemann's ester 进行 Michael 加成得到产物 19.19 被 $\mathrm{LDA}$ 剥离羰基的 $\alpha-\mathrm{H}$, 与 $\mathrm{ZnCl}_{2}$ 形成烯醇锌中间体，接着与取代的丙酤发生反应得到 aldol 产物 20. 20 再在 $p$ - $\mathrm{TsOH}$ 条件下回流环化成呋喃化 合物 21. 接下来就是构建 2 的 3/5 并环, 将酯基水解、 与甲基锂反应得到甲基酮 22.22 再选择性去质子化，与

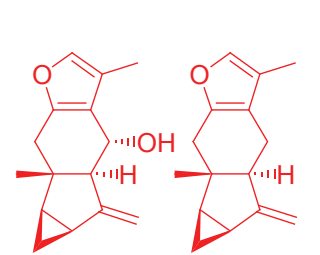

lindene 1

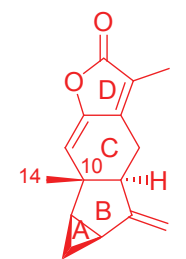

lindene 2 chloranthalactone $\mathrm{A}=$

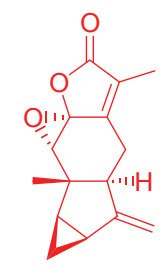

chloranthalactone B (4)

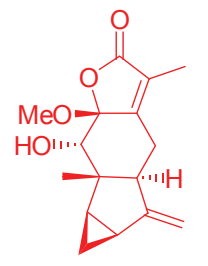

9-hydroxy heterogorgiolide (5)
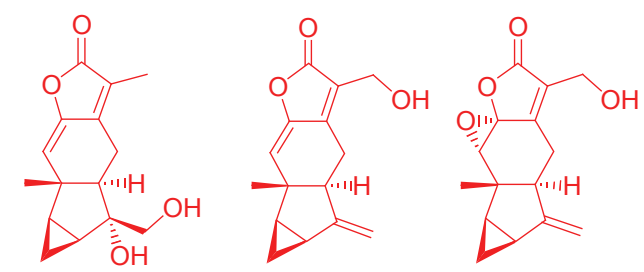

shizukanolide E(6) onoseriolide (7) oxyonoseriolide (8)

Chart 1

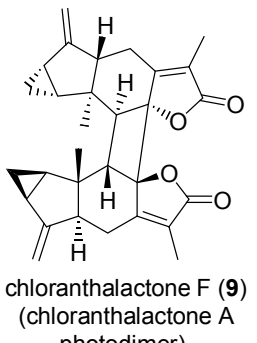
photodimer)

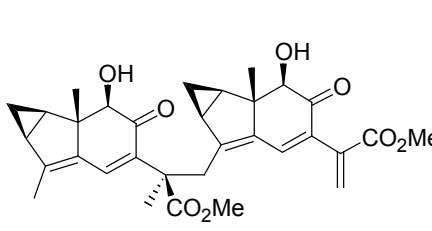

shizukaol J (14)

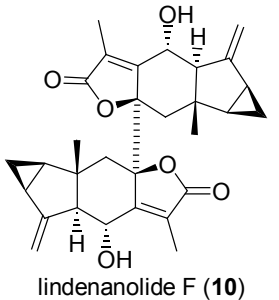

indenanolide $F(10)$

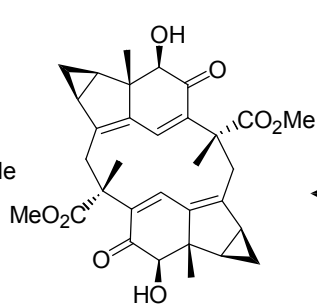

cycloshizukaol (15)

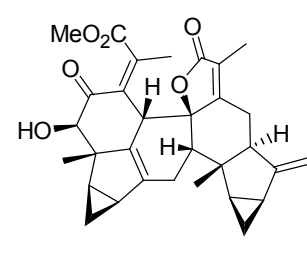

shizukaol A (11)

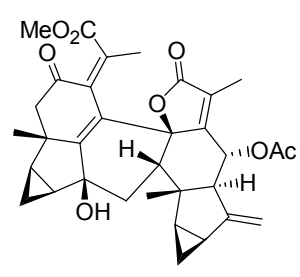

chlorahololide A (12)

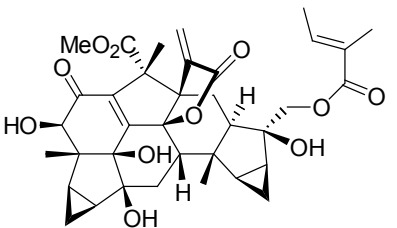

sarcanolide A (13)

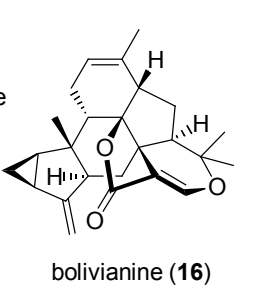

bolivianine (16)
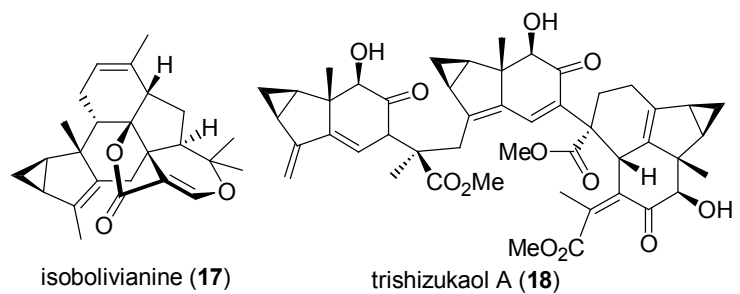

Chart 2 


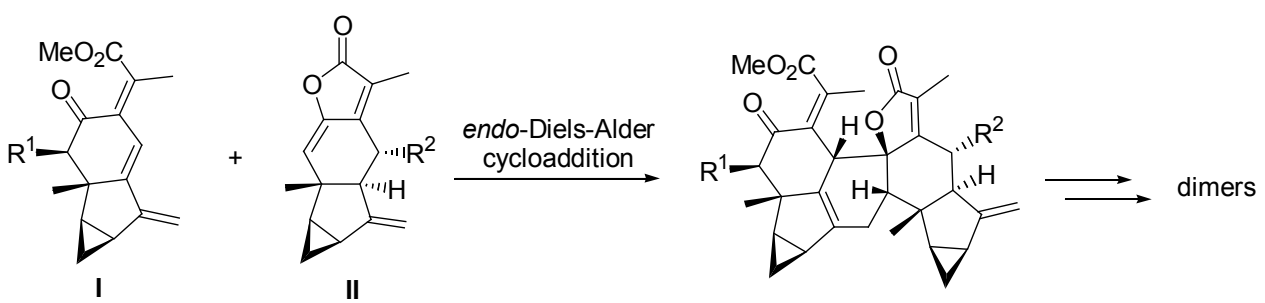

Scheme 1

三氟乙酸三氟乙基酯反应得到二酮。在碱性条件下，二 酮与 $\mathrm{TsN}_{3}$ 作用形成重氮化合物，即关键反应的前体 23 . 获得关键反应的前体后，作者利用催化剂 $\mathrm{Rh}_{2}(\mathrm{OAc})_{4}$ 或 $\mathrm{Cu}(\mathrm{acac})_{2}$ 尝试了关键反应, 并进行了优化, 最后在 $\mathrm{Cu}(\mathrm{acac})_{2}$ 及甲苯加热的条件下发生分子内的插入, 高非 对映选择性地得到 $c i s, c i s-3 / 5 / 6$ 三环产物 25 和 cis,trans-3/5/6 三环产物 26, 而没有生成正确构型的 cis, trans-3/5/6 三环产物 24. 究其原因可能是在关键反应 条件下, 由于 trans-5/6 并环结构不稳定, 即使在中性条 件下也易异构化为更稳定的 cis-5/6 并环结构的化合物 [30]. 最后 25 和 26 的混合物再经过 Julia-Kocienski 亚甲 基化反应得到化合物 2 的差向异构体 $( \pm)$-epi-lindenene (27), 以及环丙基与甲基处于 $\mathrm{B}$ 环异侧的 cis-5/6 并环产 物 $( \pm)$-iso-lindenene (28). 从以上结果, 我们可以看出: 当 $\mathrm{B}$ 环上的环丙烷与甲基处于同侧时，由于位阻的增 大，使得原本不太稳定的 trans-5/6 并环关键中间体在中 性条件迅速地异构化为 $c i s-5 / 6$ 并环产物, 而在 iso-lindenene 中, 环丙烷与甲基处于 $\mathrm{B}$ 环的异侧使得相 应的关键中间体张力大大减小, 这可能是化合物 28 能
稳定存在的关键. 该论文发表至今，未见作者后续的研 究工作报道.

\section{2 南发俊小组}

2010 年,南发俊课题组 ${ }^{[31]}$ 报道了对二聚体 chlorahololide A (12)的全合成研究，他们通过 1,6-烯炔还原环 化及分子内 Heck 反应为关键策略来构建 chlorahololide $\mathrm{A}$ 的三烯片段(Scheme 3). 以 3/5 并环内酯 29 为原料, 首 先用甲基锂对其内酯进攻，开环形成甲基酮，接着将新 生成的羟基用 TBS 保护得到化合物 30. 30 再与二乙基 膦酸酯进行霍纳尔-沃兹沃思-埃蒙斯(HWE) 反应得到 (E)-产物 31. 为了构建端炔，他们将 31 差基上的 TBS 去 掉、Swern 条件下氧化成醛 32, 醛再被 TMS 乙炔锂加 成得到炔醇. 接着为了得到 1,6-烯炔关键中间体，作者 将炔醇炔丙位的羟基用 DHP 进行保护，脱除端炔的保 护基 TMS 转化为化合物 33. 再还原 33 的酯基得到伯醇, 最后伯醇用 TBS 保护形成了关键中间体 1,6-烯炔 34. 接 下来进行钯催化的还原环异构化的反应，高产率地形成 如图所示的产物 35. 化合物 35 脱除 TBS、氧化形成醛

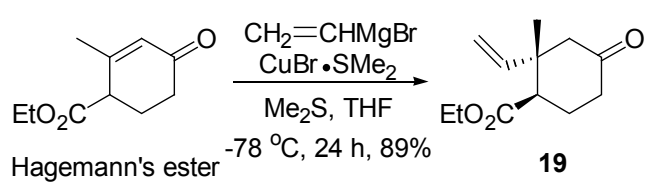

19

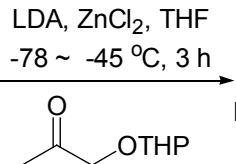<smiles>C=C[C@]1(C)CC(=O)C(C(C)(O)C[OH2+])CC1C(=O)OCC</smiles>

20

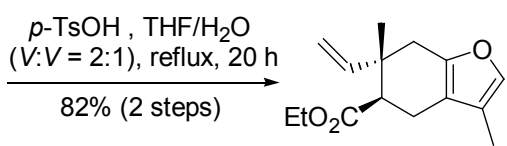

21

(1) $\mathrm{LiOH} \cdot \mathrm{H}_{2} \mathrm{O}, \mathrm{H}_{2} \mathrm{O}$, dioxane $100{ }^{\circ} \mathrm{C}, 16 \mathrm{~h}, 90 \%$ (2) $\mathrm{MeLi}, \mathrm{Et}_{2} \mathrm{O}, 0^{\circ} \mathrm{C}$ $2 \mathrm{~h}, 72 \%$

LiHMDS, THF, then<smiles>Cc1coc2c1CC1C(=O)C3C[C@H]3C1(C)C2</smiles>
25<smiles>Cc1coc2c1C[C@H]1C(=O)[C@@H]3C[C@H]3[C@]1(C)C2</smiles>
\begin{tabular}{c}
$\mathrm{Cu}(\mathrm{acac})_{2}, \mathrm{PhH}$ \\
\hline $50^{\circ} \mathrm{C}, 55 \%$ \\
$d r=20: 1$
\end{tabular} 26<smiles>C=C[C@]1(C)Cc2occ(C)c2C[C@H]1C(=O)C=N</smiles>
23

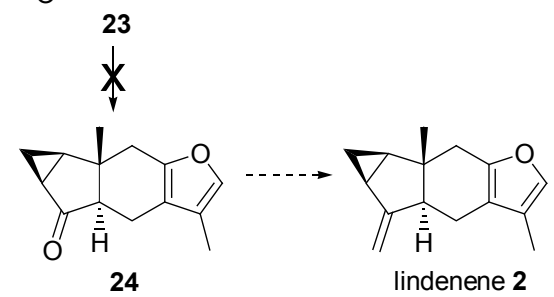<smiles>C=C[C@]1(C)Cc2occ(C)c2C[C@H]1C(C)=O</smiles>

22

Scheme 2 
36, 36 再与格氏试剂 37 加成得到 38 和 39, 化合物 39 脱除保护基 THP 及氧化得到 Heck 反应的前体 40, 最后 40 经过 endo-Heck 反应及 Julia-Kocienski 亚甲基化构建 了三烯片段 41.

\section{3 赵刚小组}

2011 年, 赵刚小组 ${ }^{[32]}$ 报道了 chlorahololide $\mathrm{A}$ 前体 49 的手性合成, 采用手性的起始原料 $(R)$-Hajos-Parrish 酮 [合成方法: 2-甲基-1,3-环戊二酮在 $L$-脯氨酸的催化下
与甲基乙烯基酮(MVK)发生 Michael 加成，接着环化], 其关键反应为 $t-\mathrm{BuCu}$ 催化的立体选择性的还原及羒基 诱导的 Simmons-Smith 环丙烷化(Scheme 4). 合成路线 为: $(R)$-Hajos-Parrish 酮经过立体选择性地还原生成热力 学不稳定的 trans $-5 / 6$ 并环产物 42, 选择性保护位阻小的 羰基形成缩酮 43. 接下来利用 Saegusa 氧化在 43 的五元 环上构建 $\alpha, \beta$-不饱和醖 44 的双键, 生成的双键再被过氧 叔丁醇立体选择性地环氧化, 然后在 $\mathrm{TMSCl}$ 存在下环

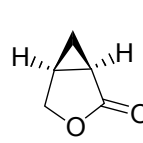

(1) MeLi, THF/Et $2 \mathrm{O}$

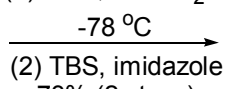

$73 \%$ (2 steps)

29

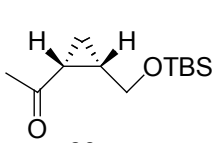

30

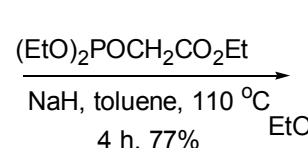

$4 \mathrm{~h}, 77 \%$
$\mathrm{EtO}_{2} \mathrm{C}_{31}^{\prime}$

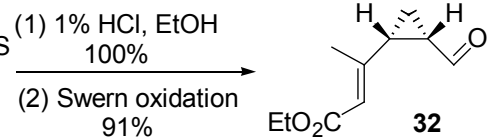

(1) TMS $=\mathrm{Li}, 99 \%$

(2) DHP, PPTS, $100 \%$

(3) TBAF, THF/ $/ \mathrm{H}_{2} \mathrm{O}, 100 \%$,<smiles>C=C1C([O-])C2CC2[C@@]1(C)CC=O</smiles>

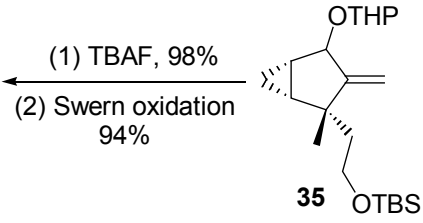

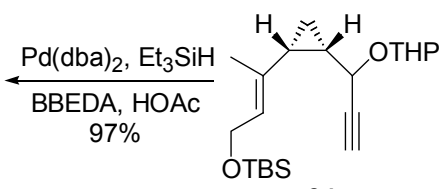

(1) DIBAL-H, toluene

(2) TBSCl, imidazole 91\% (2 steps) $\quad \mathrm{EtO}_{2} \mathrm{C}^{-}$<smiles>C#CC([OH+])[C@H](C)[C@H](C)/C(C)=C/C(=O)OCC</smiles>
34

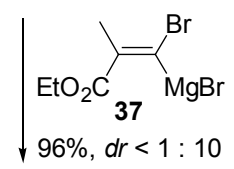

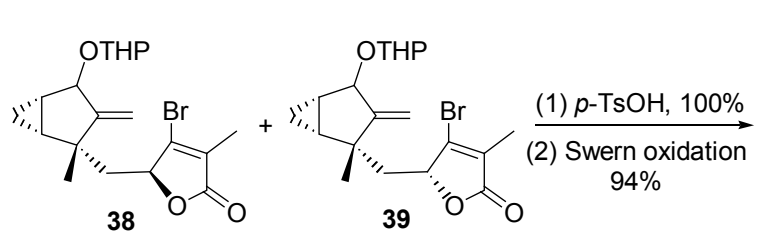

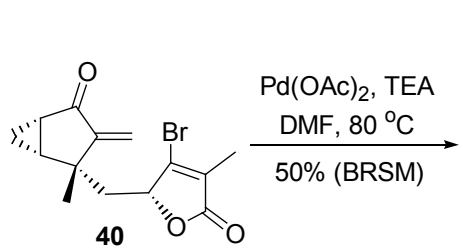

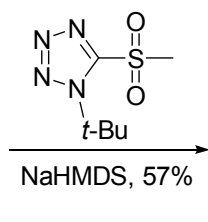

40

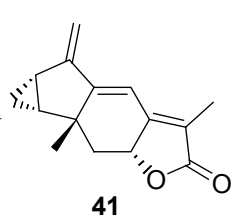

Scheme 3

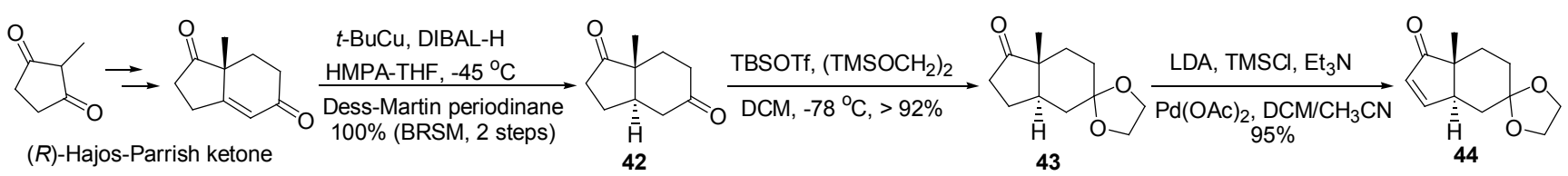

(1) LDA, TMSCl, $\mathrm{Et}_{3} \mathrm{~N}$; $m$-CPBA, $\mathrm{NaHCO}_{3}, \mathrm{CH}_{2} \mathrm{Cl}_{2}, 0^{\circ} \mathrm{C}$; TBAF, THF, $0^{\circ} \mathrm{C}$

(1) TBHP, DBU, DCM r.t., $30 \mathrm{~h}, 85 \%$

(2) $\mathrm{N}_{2} \mathrm{H}_{4} \cdot \mathrm{H}_{2} \mathrm{O}, \mathrm{TMSCl}$

(2) $\mathrm{TsNH}_{2} \mathrm{NH}_{2}$, EtOH, r.t.; DMF, r.t., 5 min, ca. $60 \%$

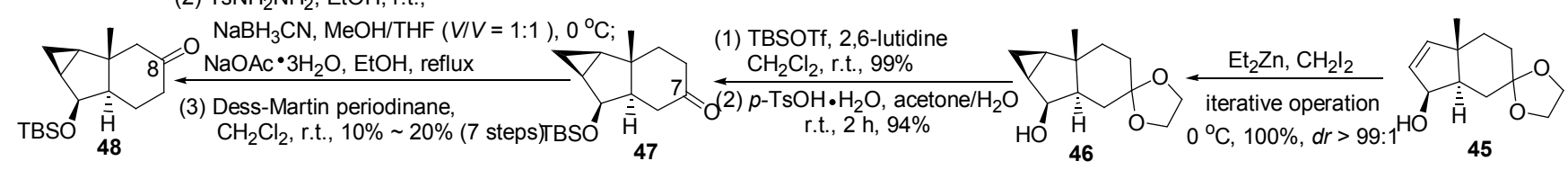

LiHMDS, methyl pyruvate $\mathrm{ZnCl}_{2}$, THF, $-78^{\circ} \mathrm{C}$;

$p$ - $\mathrm{TsOH} \bullet \mathrm{H}_{2} \mathrm{O}$, benzene reflux, $20 \mathrm{~min}, 80 \%$ (2 steps)

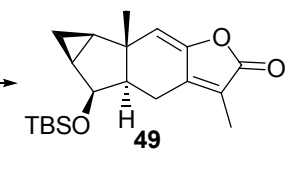

Scheme 4 
氧开环，水合肼对环氧化合物进行羰基还原形成烯丙醇 得到 cis,trans-3/5/6 三环产物 46, 化合物 46 的差基被 TBS 保护，脱除乙二醇保护基后，7-位的酮羰基经过 7 步 转位得到 8-位的羰基化合物 48. 最后在 $\mathrm{LiHMDS} / \mathrm{ZnCl}_{2}$ 条件下 48 与丙酮酸甲酯发生 aldol 反应，接着在对甲苯 磺酸条件下加热回流形成丁烯内酯环的产物 49, 即 chlorahololide A 的前体.

随后在 2012 年, 他们发表了(一)-lindene (2), (十)金粟兰内酯 $\mathrm{A}(3)$ 及 $\mathrm{F}(9)$ 的全合成工作 $(\text { Scheme } 5)^{[33]}$, 采 用已报道的中间体 46 出发. 将其中的羟基氧化、 Julia-Kocienski 亚甲基化形成环外烯烃, 接着在 $\mathrm{FeCl}_{3} \cdot \mathrm{SiO}_{2}$ 作用下脱除乙二醇保护基得到酮 50. 值得注 意的是, 此时 trans-5/6 并环未发生异构化为 cis-5/6 并 环. 为了缩短步骤, 他们尝试直接对 C-8 进行氧化, 在 $\mathrm{NaH}$ 存在下 50 与膦酸酯发生霍纳尔-沃兹沃思-埃蒙 斯(HWE)反应得到混合物 $\mathbf{5 1}, E / Z=1.3: 1$. 为了在 C-8 上直接引入羟基, 他们尝试了大量的条件, 最后发现在 $\mathrm{CrO}_{3} / 3,5$-二甲基吡唑/DCM 的条件下回流得到单一构型 的内酯产物 52, 高立体选择性的可能原因在于 C-8 的 $\mathrm{a}-\mathrm{H}$ 相对 e-H 有较大的位阻(C-10 的甲基), 不易被氧化. 但一步区域选择性及立体选择性的引入烯丙位羟基，再 发生内酯环化的串联反应很有趣. 随后 DIBAL-H 还原 52 为半缩醛 53, 53 在 PPTS/DDQ 条件下, 先形成呋喃环 的产物(一)-lindene (2), 2 的呋喃环再被 DDQ 氧化为 $\gamma$ 丁烯内酯得到 $(+)$-金粟兰内酯 A (3). 作者还发现直接 用 DDQ 也可以将 $\mathbf{5 3}$ 直接氧化为 $\mathbf{3}$, 产率大为提高. 最 后 $(+)$-金粟兰内酯 $\mathrm{A}$ 在高压永灯光照下高效转化为 $(+)$-金粟兰内酯 F (9).

\section{4 彭小水小组}

在赵刚小组 ${ }^{[34]} 2011$ 年相关工作发表后不久, 彭小
45. 烯丙位羟基诱导的 Simmons-Smith 环丙烷化 水小组也发表了关于 chlorahololide A 的合成研究 (Scheme 6). 他们的研究思路是先进行 Diels-Alder 反应 构建连接倍半萜二聚体两个片段的六元环骨架，再来修 饰外围的结构. 其关键步骤为分子内的阴离子 $\mathrm{S}_{\mathrm{N}} 2$ 环化 反应及 Diels-Alder 反应，合成从 $( \pm)$-Wieland-Miescher 酮开始，经 $\mathrm{NaBH}_{4}$ 选择性还原、 $\mathrm{Ms}$ 保护得甲磺酸酯 $\mathbf{5 4}$, 接着 $\alpha, \beta$-不饱和酮烯醇硅醚化、 $\mathrm{Ac}$ 交换 $\mathrm{TBS}$ 形成醋酸 酯 55. $m-\mathrm{CPBA}$ 氧化 $\mathbf{5 5}$ 中远离 $\mathrm{Ac}$ 的双键，同时 $\mathrm{Ac}$ 解离 成酮，再在弱碱性条件下发生分子内环氧开环得到混合 物 56, $\alpha: \beta=1: 3$. 将化合物 56a 的 $\alpha-\mathrm{OH}$ 用 Ac 保护得 到 57; 化合物 56b 的 $\beta-\mathrm{OH}$ 经 Mitsunobu 反应，构型翻 转也得到 57. 此时需将烯酮的羰基转化为缩酮或硫缩 酮而保护，然而他们尝试了多个条件均未如愿. 随后他 们改变策略, 采用迁回的方式先将 57 还原为醇, 再用 TBS 将其差基保护起来得到 58. 化合物 58 经脱 Ac, Dess-Martin氧化即得到进行 $\mathrm{S}_{\mathrm{N}} 2$ 反应的前体 59. 化合物 59 在 KHMDS 作用下篗取羰基 $\alpha-\mathrm{H}$, 立即发生分子内 $\mathrm{S}_{\mathrm{N}} 2$ 反应得到 $3 / 5 / 6$ 三环产物 60. 为了得到 Diels-Alder 反应的前体双烯 $62, \mathrm{MeLi}$ 对化合物 60 的酮羰基加成, 再用 Martin 试剂消除叔醇成端烯. 而为了得到亲双烯体 67, 化合物 61 脱除 TBS、氧化、烯醇硅醚化及 Saegusa 条件下剥掉 $\beta$ - $\mathrm{H}$ 形成亲双烯体二烯酥 67 . 亲双烯体 65 也可以通过类似的过程得到，化合物 60 先将羟基还原、 $\mathrm{Ac}$ 保护得到化合物 63. 接着除去 63 的另一个羟基上的 TBS、氧化形成酮 64，该化合物脱除 Ac，羟基及羰基均 接上 TBS, 最后在 Saegusa 条件下烯醇硅醚形成另一个 双键，完成了 65 的合成.

随后他们进行了两个片段 62 与 65(或 67)的 DielsAlder 反应研究(Scheme 7), 在甲苯作溶剂、封管及加热

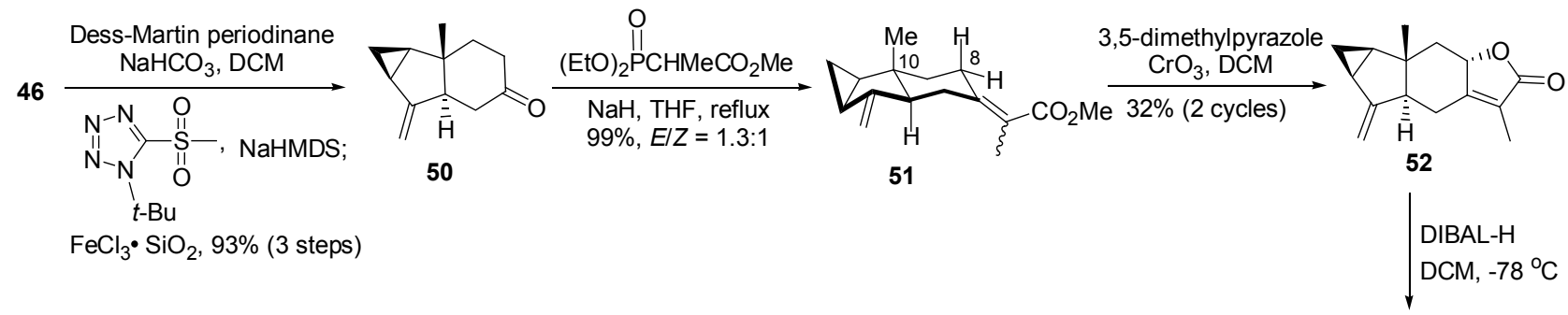

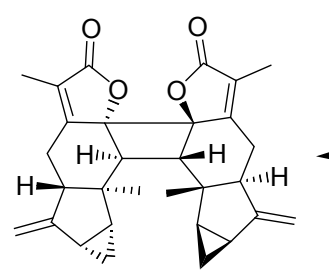

(+)-chloranthalactone F (9)

DDQ, DCM, 80\%

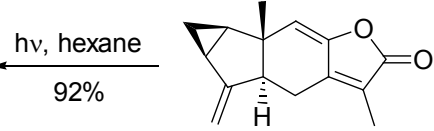

(+)-chloranthalactone A (3)

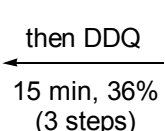

(3 steps)

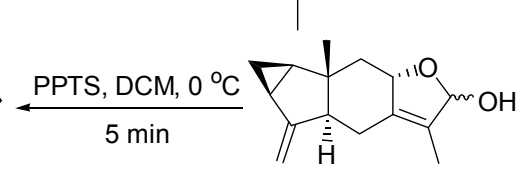

53

Scheme 5 


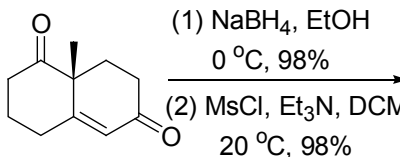

$( \pm)$-WielandMiescher Ketone

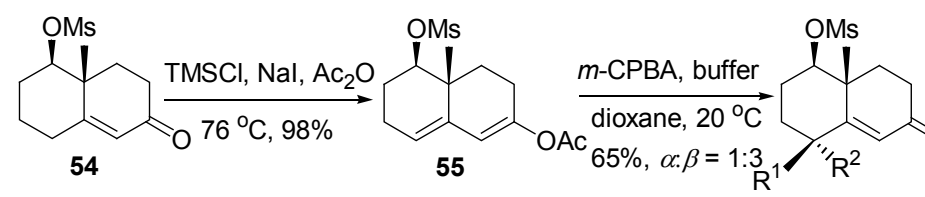

(1) $\mathrm{NaBH}_{4}, \mathrm{CeCl}_{3} \cdot 7 \mathrm{H}_{2} \mathrm{O}$

$-78^{\circ} \mathrm{C}$ (2) TBSCl, imidazole

${ }^{O}$ DMAP, DMF, $20{ }^{\circ} \mathrm{C}$ $\alpha: \beta=1: 19,92 \%$ (2 steps)

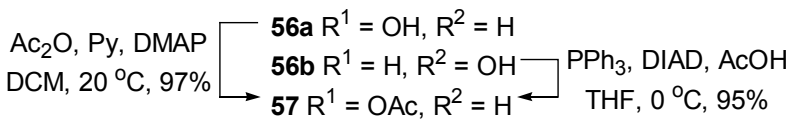<smiles>C=C1C2=C[C@@H]([OH+])CC[C@]2(C)[C@@H]2C[C@H]12</smiles>

Martin's sulfurane $\mathrm{DCM}, 0{ }^{\circ} \mathrm{C}, 96 \%$
OTBS (2) $\begin{gathered}\text { Dess-Martin periodinane } \\ \mathrm{NaHCO}_{3}, \mathrm{DCM}, 20^{\circ} \mathrm{C}, 95 \%\end{gathered}$ 58

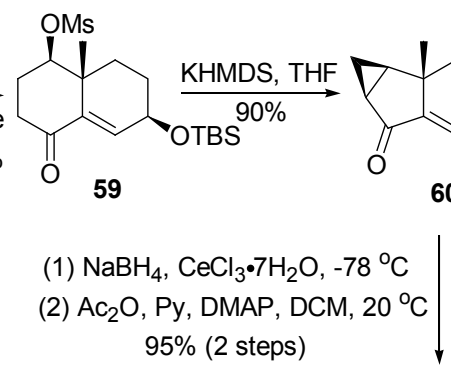

(1) $\mathrm{K}_{2} \mathrm{CO}_{3}, \mathrm{MeOH}$ $\mathrm{THF}, 20^{\circ} \mathrm{C}$

(2) TBSOTf, $\mathrm{Et}_{3} \mathrm{~N}$<smiles>C=C1C=CC2(C)C(=C1)CC1CC12</smiles>

TBSO 65
DCM, $-78^{\circ} \mathrm{C}$

$\mathrm{O}$ (3) $\mathrm{Pd}(\mathrm{OAc})_{2}$ $\mathrm{CH}_{3} \mathrm{CN}, 20^{\circ} \mathrm{C} \quad \mathrm{AcO}$ $73 \%$ (3 steps)

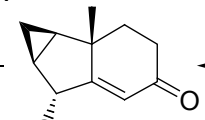

64

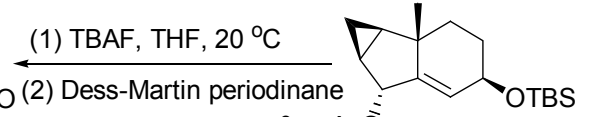
$\mathrm{NaHCO}_{3}, \mathrm{DCM}, 20^{\circ} \mathrm{C} \quad \mathrm{AcO}$ 91\% (2 steps)

63
(1) TBSOTf, $\mathrm{Et}_{3} \mathrm{~N}$

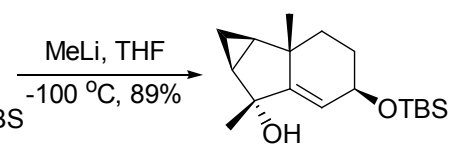

61

(1) TBAF, THF, $20{ }^{\circ} \mathrm{C}$

(2) Dess-Martin periodinane $\mathrm{NaHCO}_{3}, \mathrm{DCM}, 20^{\circ} \mathrm{C}$ $84 \%$ (2 steps)

(2) $\mathrm{Pd}(\mathrm{OAc})_{2}, \mathrm{CH}_{3} \mathrm{CN}$ $20^{\circ} \mathrm{C}, 78 \%$ (2 steps)<smiles>C[C@@]1(O)C2=CC(=O)C=C[C@]2(C)[C@@H]2CC21</smiles>

Scheme 6

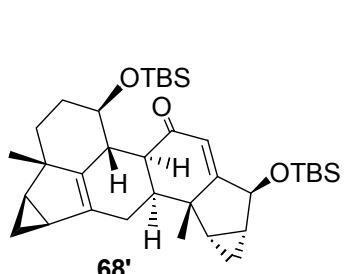

68'

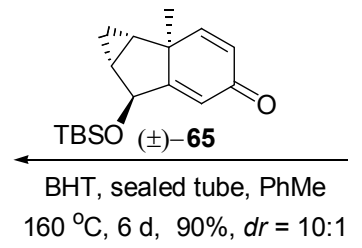

$160{ }^{\circ} \mathrm{C}, 6 \mathrm{~d}, 90 \%, d r=10: 1$

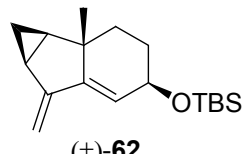

$( \pm)-62$
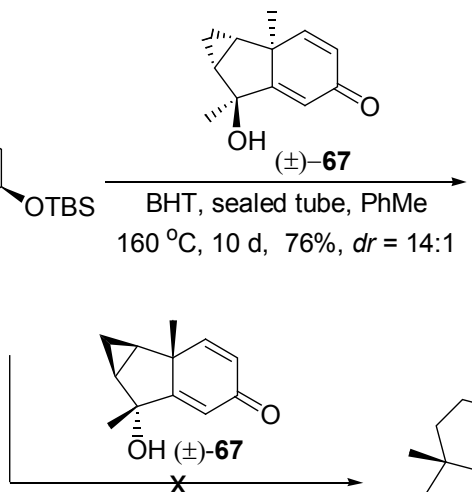

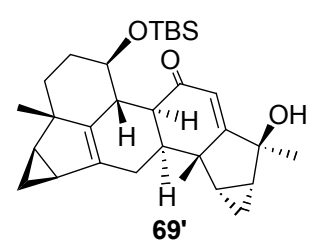

$69^{\prime}$

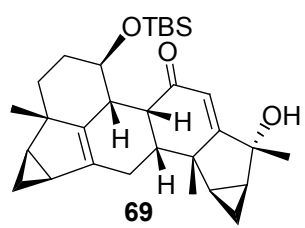

Scheme 7

的条件下以高非对映选择性分别得到两个 Diels-Alder 主产物 68'和 69'. 仔细分析 69'的单晶结构, 不难看出产
物都是双烯体与亲双烯体的 exo-产物，可能原因在于 exo-Diels-Alder 反应使它们的空间位阻最小. 


\section{5 刘波小组}

1.5.1（士)-金栗兰内酯 A (3) 和 $( \pm)$-银线草内酯 E (6) 的第一代合成研究

我们小组 ${ }^{[35]}$ 也对乌药烷型倍半萜进行合成的研究, 我们的目标是先合成结构较为简单的倍半萜单体, 然后 在此基础上实现二聚体的全合成. 合成策略是利用 Hodgson 环丙烷化构建乌药烷型倍半萜 cis,trans-3/5/6 骨 架(Scheme 8). 首先进行了模板反应的研究, Hagemann's ester 经过乙烯基酮试剂的 Michael 加成、酮羰基被乙二 醇保护得到已知化合物 70, 70 再经还原、氧化得到醛 71 . 在 $\mathrm{Me}_{3} \mathrm{~S}^{+} \mathrm{I}^{-} / \mathrm{NaH} / \mathrm{DMSO}$ 的条件下, 71 迅速地得到环氧的 混合物 72a 及 72b. 接下来尝试在 Hodgson 的条件下 72a 及 72b 的环丙烷化反应, 令人欣喜的是, 在 $\mathrm{LiTMP} / \mathrm{Et}_{2} \mathrm{O}$ 条件下, 环氧混合物顺利发生环化得到两种可分离的产 物 73a 和 73b, 两者比例约为 $4: 1$. 相应的立体化学由 脱除保护基产物的 NOE (nuclear overhauser effect)谱进 行确认，这表明利用六元环的椅式构象来控制反应的立 体选择性是高效可行的.

有了这些结果后, 接下来的工作就是构建环外双 键. 易于考虑到的方式是经酮羰基, 再烯化来构建, 但 5/6 并环体系的顺反异构化, 显然有很强的底物依赖性. 首先我们考察了 $73 \mathrm{~b}$ 经氧化成酮后发生异构化, Dess-Martin 氧化得到酮 75, 但从 ${ }^{1} \mathrm{H}$ NMR 上不能判断 异构体的存在, 也无法断定是否完全异构化为顺式的产 物. 因此我们将 72 在 $\mathrm{NaBH}_{4} / \mathrm{MeOH}$ 条件下还原, 经柱 层析分离得到四个异构体 73b, 73c, 73d, 73e, 产率分别 为 $12.6 \%, 33 \%, 29.9 \%, 2.2 \%$. 73c 的羟基用 TBS 保护, 再脱除乙二醇保护基, 得到的酮 76 与赵刚小组 ${ }^{[2]}$ 报道 的化合物 48一致; 而 73d 脱除乙二醇后的产物经 DEPT, HMQC 及 NOE 谱确认无误为 $c i s-5 / 6$ 并环产物 77. 由此

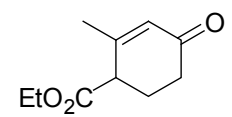

Hagemann's ester
(1) vinyl $\mathrm{MgBr}, \mathrm{CuBr} \cdot \mathrm{Me}_{2} \mathrm{~S}$

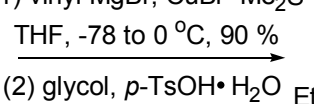

$\mathrm{PhH}$, reflux, 93\%

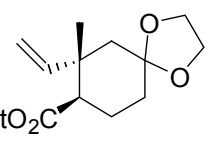

70

推断 75a/75b trans/cis) 比例约为 $1.4 ： 1$, 可见结构上的 差异并非如 Bladwin 所观察到的结果一样完全异构化, 而是存在一种平衡关系(Scheme 9). 此时我们认为用 Wittig 或 Julia 烯化反应引入亚甲基显得效率比较低, 在 碱性条件下可能会进一步消旋化，而且两种产物很可能 不好分离.

为了能有效地引入亚甲基，我们小组也考虑过首先 先在羟基上引入离去基团, 然后发生 $\mathrm{S}_{\mathrm{N}} 2$ 反应引入一个 或两个碳, 再转化为环外双键. 在羟基上首先引入好的 离去基团 $\mathrm{Tf}$, 然而在 $\mathrm{Tf}_{2} \mathrm{O} / 2$,6-lutidine 或 $\mathrm{Tf}_{2} \mathrm{NPh} / \mathrm{DMAP}$ 条件下却得到无法分离的混合物, 可能是因为环丙烷的 存在, 羟基上一旦引入 $\mathrm{Tf}$ 后，极易发生重排反应. 随后 选择引入离去能力稍差的 $\mathrm{Ms}$, 以 $83 \%$ 的收率得到产物 78, 然而尝试引入小位阻的 “ $\mathrm{CN}$ ” 或乙炔基等基团时, 均未成功(Scheme 10). 这可能由于底物的位阻太大, 阻 碍亲核取代反应的进行.

我们转而考虑更为直接简洁的策略, 在关键反应之 前引入一个碳原子, 即使用偕二取代的环氧化合物为关 环前体构建并环骨架(Scheme 11). 化合物 70 经水解、 甲基锂加成得到甲基酮 80 , 再通过三步连续的操作得 到 $\alpha$-羟基酮 81, 即: 甲基酮形成 TMS 的烯醇硅醚，接着 区域选择性地环氧化烯醇硅醚, 最后 TBAF 脱除 TMS, 环氧开环，三步收率为 $68 \%$. 首先我们在 $\mathbf{8 1}$ 的羟基上引 入 MOM 基团, 再环氧化. 虽然在 $\mathrm{Me}_{3} \mathrm{~S}^{+} \mathrm{I}^{-} / n-\mathrm{BuLi}$ 条件 下其羰基被环氧化能得到预料的环氧化产物，但同时伴 随许多 $\alpha^{\prime}$ 位消旋的环氧化产物, 两者产率接近 $1: 1$. 为 了防止酮的 $\alpha^{\prime}$ 位消旋，利用大位阻保护基获得更好的底 物构象控制, 选用了 TBS 来代替 MOM. 在经典条件下 处理 81 得到 TBS 保护的羟基酮，接着 $\mathrm{Me}_{3} \mathrm{~S}^{+} \mathrm{I}^{-} / n-\mathrm{BuLi}$ 下羰基被环氧化为 $\mathbf{8 1}$, 同时生成少量可分离的 $\alpha^{\prime}$ 位异构

(1) DIBAL-H, DCM

$0{ }^{\circ} \mathrm{C}$ to r.t., $86 \%$

(2) Swern oxidation, DCM $-78{ }^{\circ} \mathrm{C}$ to r.t., $95 \%$

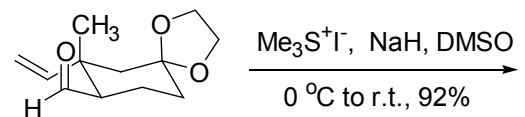

71
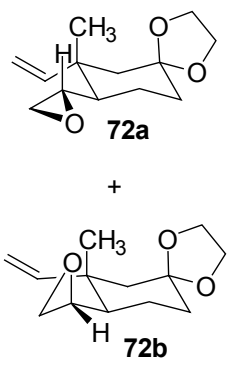

$72 a / 72 b=4 / 1$, mixture

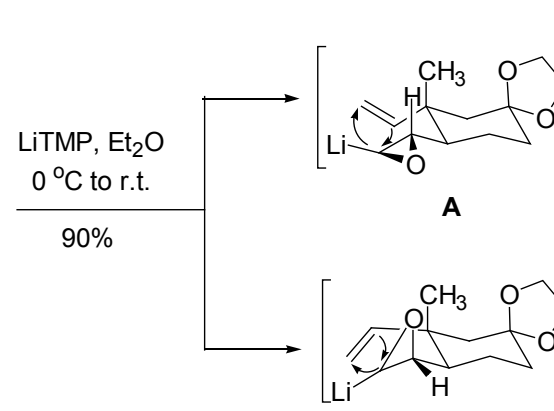

B
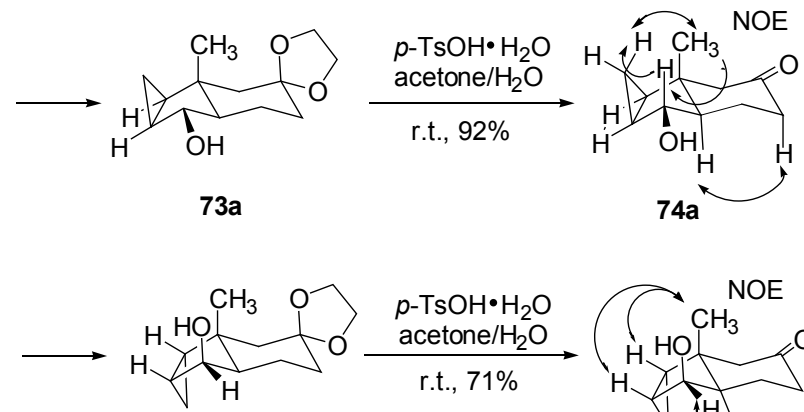

73b

$73 a / 73 b=4 / 1$, seperable

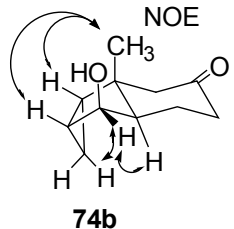

Scheme 8 


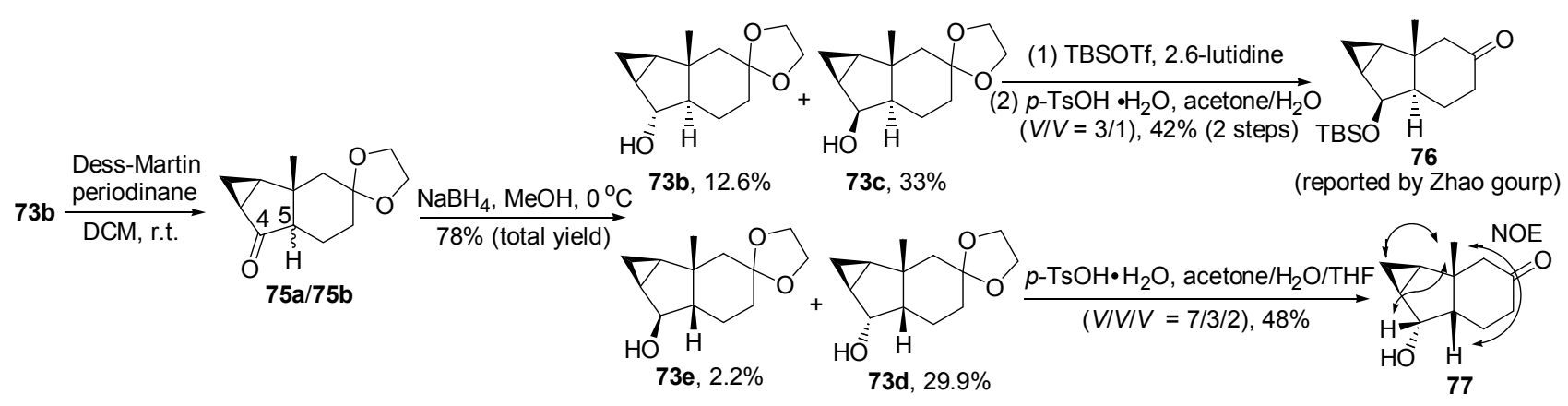

Scheme 9

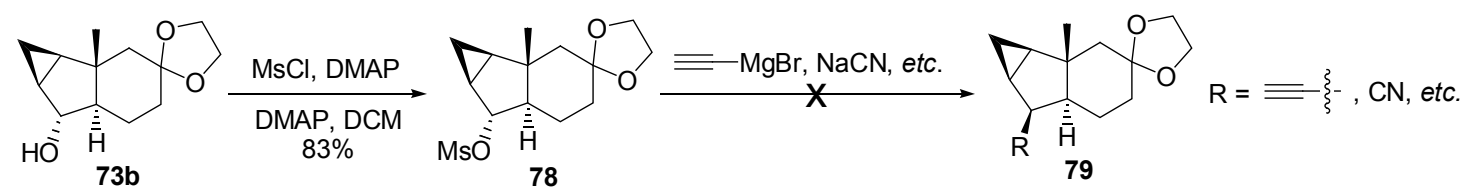

Scheme 10

化的产物. 由于前期关键反应研究中 TBS 作保护基的 底物比 MOM 的副反应更复杂 ${ }^{[36]}$, 因而将 82 转化为 MOM 保护的化合物 83. 环氧化合物 83 在 LiTMP/ $t$-BuOMe 及 $0{ }^{\circ} \mathrm{C}$ 的条件下环化得到两个三环产物 84 和 85, 约为 $8.4: 1$. 值得注意的是, 这种类型的偕二取代 环氧化物的成功转化拓展了 Hodgson 反应的底物适用 范围. 主产物 84 在浓度为 $6 \mathrm{~mol} / \mathrm{L}$ 的盐酸中同时脱除乙 二醇及 MOM 保护基形成二醇 86, 后者在硫代羰基二咪 唑、DMAP 存在下顺利生成硫代碳酸酯 87, 经 X-ray 证 实其相对立体构型. 其后就是构建金粟兰内酯 $\mathrm{A}$ 的 $\mathrm{D}$ 环. 在 $\mathrm{LDA}$ 及 $\mathrm{ZnCl}_{2}$ 作用下, 87 与丙酮酸乙酯发生 aldol 反应, 得到 $\gamma$-羰基酯 $\mathbf{8 8}$. 由于硫代碳酸酯基团在强碱下 不太稳定，在最佳条件下收率也仅仅只有 57\% (84\%
BRSM). 而此时若脱除硫代碳酸酯成环外双键，则随后 在酸性条件环化时，双键易于发生异构化形成环内的双 键 $^{[6 b, 7 a]}$. 最后 88 在酸性条件下环化及脱除硫代碳酸酯 成双键，以两步 $28 \%$ 的收率得到了天然产物 $( \pm)$-金粟兰 内酯 A (3). 硫代碳酸酯基团在酸性条件下回流会严重 分解，而尝试了其他的条件也没有改进.

同时, 我们实现了从二醇 86 出发来合成 $( \pm)$-银线 草内酯 E (6). 将二醇用亚异丙酤保护得到 89, 再进行 aldol 反应得到产物 90. 与 $( \pm)$-chloranthalactone A 的第 一代合成相似，亚异丙酮的存在使得 90 环化时也易分 解, 得到的产物再在 $-78{ }^{\circ} \mathrm{C} / \mathrm{BF}_{3} \cdot \mathrm{Et}_{2} \mathrm{O}$ 条件下与过量的 丙二硫醇发生反应, 以两步 $12 \%$ 的收率得到白色固体 (士)-6 (Scheme 12).

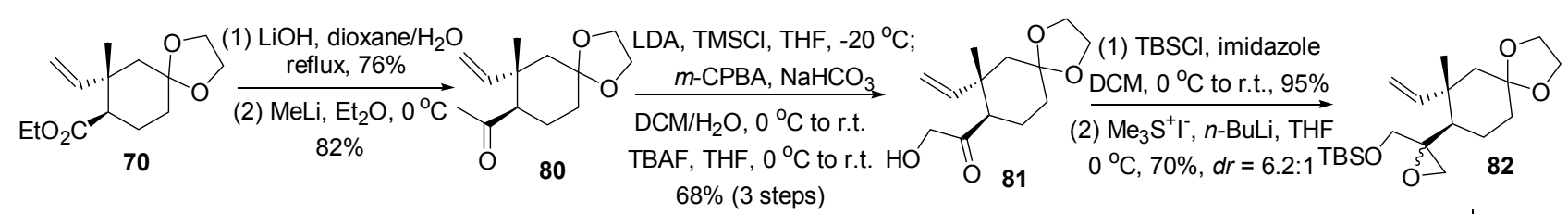

(1) TBAF, THF, r.t., $93 \%, d r=7.4: 1$ (2) $\mathrm{MOMCl}, i-\mathrm{Pr}_{2} \mathrm{EtN}, \mathrm{DCM}, 0^{\circ} \mathrm{C}$ to r.t.

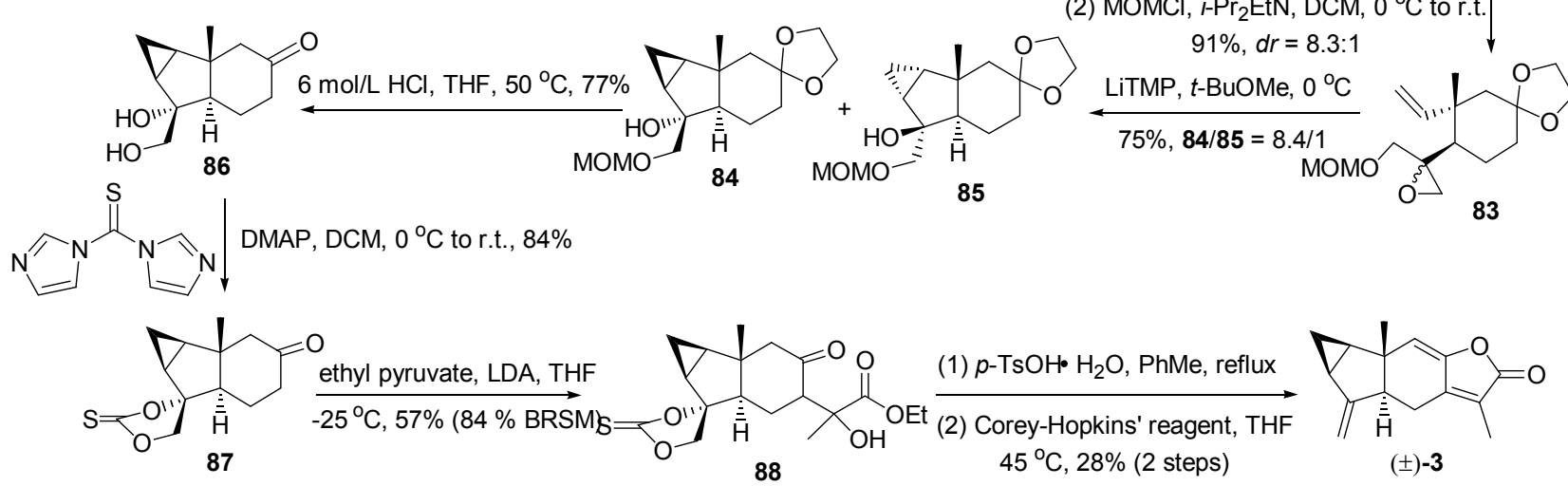

Scheme 11 


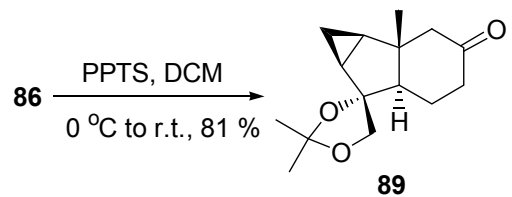

89

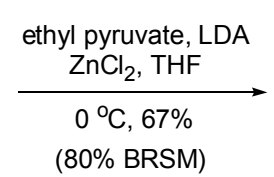

(80\% BRSM)

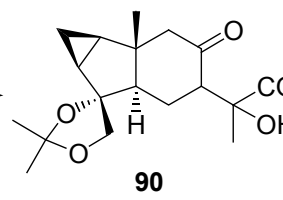

90

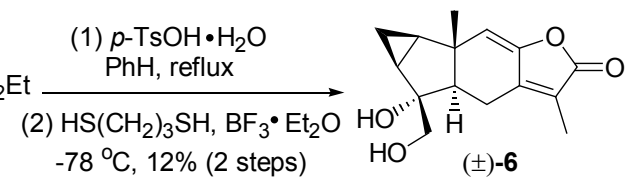

$-78{ }^{\circ} \mathrm{C}, 12 \%$ (2 steps)
$( \pm)-6$

\section{Scheme 12}

1.5.2（士)-金粟兰内酯 A (3) 和 $( \pm)$-银线草内酯 E (6) 的第二代合成研究

$( \pm)$-金粟兰内酯 $\mathrm{A}$ 和 $( \pm)$-银线草内酯 $\mathrm{E}$ 的第一代 合成路线中, 有几个比较突出的问题亟需解决: (1)环氧 化的选择性有待提高; (2)相应的环化反应的选择性也有 待改进; (3)底物 87 中硫代碳酸酯的不稳定导致 aldol 反 应时效率低; (4) $\gamma$-丁烯内酯环形成的条件有待改进; (5) 路线相对较长, 总产率较低.

基于此, 我们重新设计、修改并缩短了步骤(Scheme 13): 在 $n-\mathrm{BuLi}$ 作用下, 酯 70 直接与 $O, N$-二甲基盐酸羟 胺反应得到 Weinreb 酰胺, 接着在 $-78{ }^{\circ} \mathrm{C}$ 下 3 equiv.的 $\mathrm{LiCH}_{2} \mathrm{OMOM}$ 对酰胺进攻以高产率生成了 MOM 保护的 酮 91. 接下来经过多次尝试发现利用 Matteson 环氧化 $\left(\mathrm{CH}_{2} \mathrm{Br}_{2} / n-\mathrm{BuLi}\right)$ 条件能有效地得到单一构型的产物 92, $d r>20: 1$, 而 92 在 $\mathrm{LiTMP} / t-\mathrm{BuOMe}$ 条件下高产率得到 了单一构型的产物 84. 按第一代合成中的方法将 84 转 化为 87 后, 87 在 $\mathrm{P}(\mathrm{OMe})_{3}$ 下回流脱除硫代碳酸酯形成环 外烯烃 93.93 此时不能在酸性条件下加热形成 $\gamma$-丁烯内
酯，因为早有文献报道会发生双键移位 ${ }^{[6 b, 7 b]}$. 我们做了 一些条件探索 ${ }^{[36]}$, 发现两步条件下 $\left(p-T s \mathrm{TH} / \mathrm{Ac}_{2} \mathrm{O}\right.$ 及 DBU/THF)能有效的实现 94 的内酯化, 这样我们就完成 了(士)-3 的第二代合成. 按照相似的合成策略，从化合 物 89 出发, 我们也完成了 $( \pm)-6$ 的第二代合成.

\subsection{3（士)-金粟兰内酯 B (4), F (9)和(士)-9-Hydroxy} heterogorgiolide (5)合成研究

合成的 $( \pm)$-金粟兰内酯 $\mathrm{A}$ 不太稳定, 它的 $\mathrm{CDCl}_{3}$ 溶 液放置在冰箱 $12 \mathrm{~d}$ 后，一部分产物的 $\gamma$-丁烯内酯自动环 氧化得到 $( \pm)$-金粟兰内酯 $\mathrm{B}(4)$, 这与文献 ${ }^{[6 g]}$ 报道的一 致: 天然产物 $(-)$-金粟兰内酯 $\mathrm{A}$ 不稳定, 同样会自动氧 化成 $(-)$-金粟兰内酯 B. 接下来 $( \pm)$-金粟兰内酯 B 在 $\mathrm{MeONa} / \mathrm{MeOH}$ 的条件下快速发生反式开环，立体专一 性地得到天然产物 $( \pm$ )-9-hydroxy heterogorgiolide (5). 另外 $( \pm)$-金粟兰内酯 $\mathrm{A}$ 经过光照, 得到光二聚体 $( \pm)$-金 粟兰内酯 $F(9)$, 有趣的是, 未分离到 $( \pm)$-金粟兰内酯 $F$ 的立体异构体(Scheme 14).

(1) $\mathrm{HNMe}(\mathrm{OMe}) \cdot \mathrm{HCl}, n$-BuLi

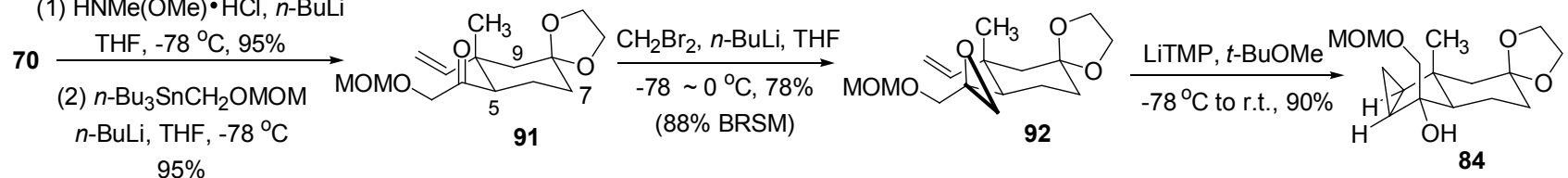
$95 \%$
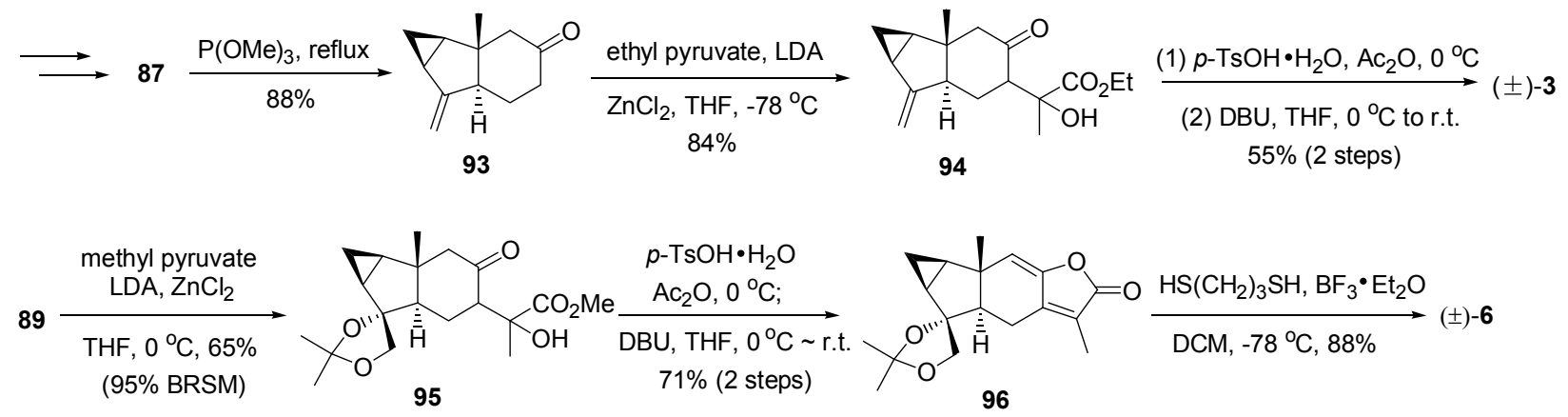

\section{Scheme 13}

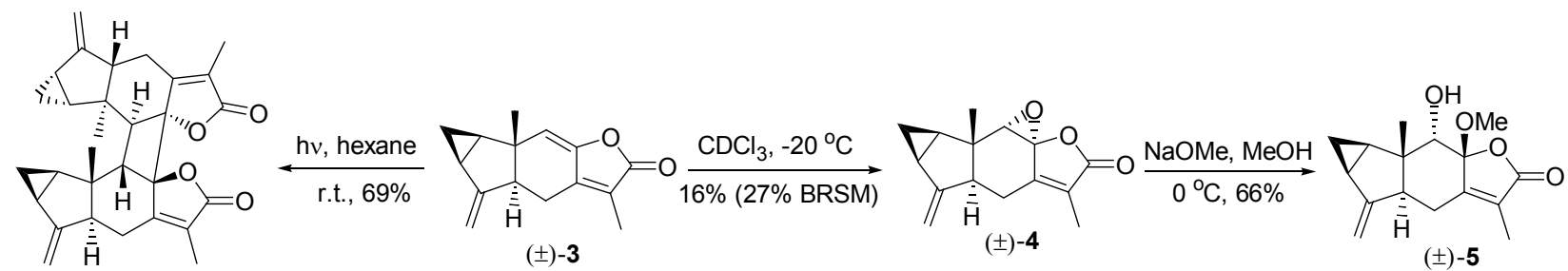
$( \pm)-9$

\section{Scheme 14}


$( \pm)$-金粟兰内酯 $\mathrm{A}$ 自动氧化形成 $( \pm)$-金粟兰内酯 $\mathrm{B}$ 的产率太低, 经过我们的努力, 最终 $( \pm)$-金粟兰内酯 $\mathrm{B}$ 的高效合成可由 (土)-银线草内酯 $\mathrm{E}$ 出发实现. 先将 (土)-6 环氧化, 再形成硫代碳酸酯, 顺利得到了化合物 97. 值得说明的是, 环氧化的产物在酸性条件下不太稳 定, 柱层析易分解. 因而反应完毕后加入三乙胺除去生 成的酸, 后处理过的粗产品不需纯化可直接用于下一 步. 最后 97 在 Corey-Hopkin 试剂下加热以定量的收率 得到了(土)-4 (Scheme 15).

$$
( \pm)-6 \frac{\text { (1) } m \text {-CPBA, } \mathrm{NaHCO}_{3}, \mathrm{DCM}, 0{ }^{\circ} \mathrm{C}}{(2) \mathrm{TCDI}, \mathrm{DMAP}, \mathrm{DCM}, \text { r.t., } 62 \% \text { (2 steps) }}
$$

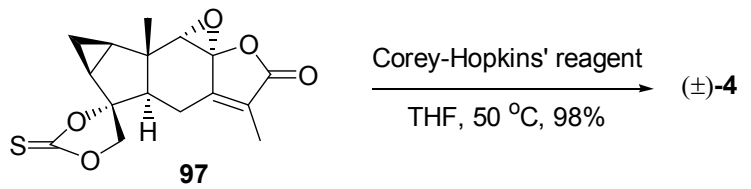

\section{Scheme 15}

\section{2 总结与展望}

迄今为止, 乌药烷型倍半萜及二聚体天然产物已分 离得到近 100 个化合物, 它们具有的 cis,trans- 3/5/6 并环 结构使得其合成具有较大挑战性. 关于它们的合成研究 文献为数不多, 也是最近几年才陆续报道的, 而其中我 国有机化学家对这类化合物的合成研究做了不少的工 作. 我们相信, 在不久的将来, 会有更多巧妙、简洁的方 法及策略被发展用于这类天然产物的合成, 以此来促进 药物化学家对该类化合物进行更为广泛的生物活性测 试与研究.

\section{References}

[1] Cao, C.-M.; Peng, Y.; Xiao, P.-G. China J. Chin. Mater. Med. 2008, 33, 1509 (in Chinese). (曹聪梅, 彭勇, 肖培根, 中国中药杂志, 2008, 33, 1509.)

[2] Cao, C. M.; Peng, Y.; Shi, Q. W.; Xiao, P. G. Chem. Biodiversity 2008, 5, 219.

[3] (a) Lian, G. Y.; Yu, B. A. Chem. Biodiv. 2010, 7, 2660. (b) Zhan, Z.-J.; Ying, Y.-M.; Ma, L.-F.; Shan, W.-G. Nat. Prod. Rep. 2011, 28, 594.

[4] Kondo, H.; Sanada, T. J. Pharm. Soc. Jpn. 1925, 45, 1047.

[5] Takeda, K.; Minato, H.; Ishikawa, M. J. Chem. Soc. C 1964, 4578.

[6] (a) Uchida, M.; Kusano, G.; Kondo, Y.; Nozoe, S. Heterocycles 1978, 9, 139.

(b) Uchida, M.; Koike, Y.; Kusano, G.; Kondo, Y.; Nozoe, S.; Kabuto, C.; Tkaemoto, T. Chem. Pharm. Bull. 1980, 28, 92.

(c) Tahara, S.; Fukiushi, Y.; Kawabata, J.; Mizutani, J. Agric. Biol. Chem. 1981, 45, 1511.

(d) Kusano, G.; Abe, M.; Koike, Y.; Uchida, M.; Nozoe, S.; Taira, Z. Yakugaku Zasshi 1991, 111, 756.

(e) Takeda, Y.; Yamashita, T.; NMatsumoto, H. Phytochemistry 1993, 33, 713 .

(f) Okamura, H.; Iwagawa, T.; Nakatani, M. Bull. Chem. Soc. Jpn. 1995, 68,3465 . (g) Tsui, W. Y.; Brown, G. D. Phytochemistry 1996, 43, 819.

(h) Tesso, H.; Konig, W. A.; Son, P. T.; Giang, P. M. Flavour Fragrance J. 2006, 21, 592.

(i) Wang, F.; Yuan, S. T.; Zhu, D. N. Chin. J. Nat. Med. 2007, 5, 174 (in Chinese).

(王菲, 袁胜涛, 朱丹妮, 中国天然药物, 2007, 5, 174.)

(j) Wu, B.; He, S.; Pan, Y. J. Tetrahedron Lett. 2007, 48, 453.

(k) Wang, X.-C.; Wu, W.-Q.; Ma, S.-P.; Liu, J.-H.; Hu, L.-H. Chin. J. Nat. Med. 2008, 6, 404.

[7] (a) Kawabata, J.; Tahara, S.; Mizutani, J.; Furusaki, A.; Hashiba, N.; Matsunoto, T. Agric. Biol. Chem. 1979, 43, 885.

(b) Kawabata, J.; Tahara, S.; Mizutani, J. Agric. Biol. Chem. 1981, $45,1447$.

(c) Kawabata, J.; Mizutani, J. Agric. Biol. Chem. 1988, 52, 2965.

(d) Kawabata, J.; Mizutani, J. Agric. Biol. Chem. 1989, 53, 203.

(e) Li, C.; Zhang, D.; Luo, Y. Acta Pharm. Sinica 2005, 40, 525 (in Chinese).

(李创军, 张东明, 罗永明, 药学学报, 2005, 40, 525.)

(f) Wang, X.-C.; Wang, L.-L.; Ouyang, X.-W.; Ma, S.-P.; Liu, J.-H.; Hu, L.-H. Helv. Chim. Acta 2009, 92, 313.

(g) Wang, C.; Zhu, L.; Yang, J.; Li, C.; Zhang, D. China J. Chin. Mater. Med. 2010, 35, 714 (in Chinese).

(王超, 朱丽萍, 杨敬芝, 李创军, 张东明, 中国中药杂志, 2010, 35, 714.)

(h) Fang, P. L.; Liu, H. Y.; Zhong, H. M. China J. Nat. Med. 2012, $10,24$.

[8] Heo, J. E.; Jin, J. L.; Lee, Y. Y.; Yun-Choi, H. S. Nat. Prod. Sci. 2005, 11,41 .

[9] Bohlmann, F.; Zdero, C.; King, R. M.; Robinson, H. Phytochemistry 1980, 19, 689.

[10] Acebey, L.; Jullian, V.; Sereno, D.; Chevalley, S.; Estevez, Y.; Moulis, C.; Beck, S.; Valentin, A.; Gimenez, A.; Sauvain, M. Planta Med. 2010, 76, 365.

[11] Wang, Q.-H.; Kuang, H.-X.; Yang, B.-Y.; Xia, Y.-G.; Wang, J.-S.; Kong, L.-Y. J. Nat. Prod. 2011, 74, 16.

[12] Zhang, C.-f.; Nakamura, N.; Tewtrakul, S.; Hattori, M.; sun, Q.-s.; Wang, Z.-t.; Fujiwara, T. Chem. Pharm. Bull. 2002, 50, 1195.

[13] (a) Bittner, M.; Jakupovic, J.; Bohlmann, F.; Silva, M. Phytochemistry 1989, 28, 271.

(b) K., T.; Horibe, I.; H., M. J. Chem. Soc. C 1968, 569.

(c) Kuang, H. X.; Xia, Y. G.; Yang, B. Y.; Wang, Q. H.; Lu, S. W. Chem. Biodiversity 2008, 5, 1736.

(d) Hu, X. R.; Yang, J. S.; Xu, X. D. Chem. Pharm. Bull. 2009, 57, 418.

(e) Kao, S.-Y.; Su, J.-H.; Hwang, T.-L.; Sheu, J.-H.; Su, Y.-D.; Lin, C.-S.; Chang, Y.-C.; Wang, W.-H.; Fang, L.-S.; Sung, P.-J. Tetrahedron 2011, 67, 7311.

(f) Kao, S.-Y.; Su, J.-H.; Hwang, T.-L.; Sheu, J.-H.; Wen, Z.-H.; Wu, Y.-C.; Sung, P.-J. Mar. Drugs 2011, 9, 1534.

[14] (a) Yang, S. P.; Gao, Z. B.; Wang, F. D.; Liao, S. G.; Chen, H. D.; Zhang, C. R.; Hu, G. Y.; Yue, J. M. Org. Lett. 2007, 9, 903.

(b) Yang, S. P.; Gao, Z. B.; Wu, Y.; Hu, G. Y.; Yue, J. M. Tetrahedron 2008, 64, 2027.

[15] (a) Kawabata, J.; Fukushi, Y.; Tahara, S.; Mizutani, J. Phytochemistry 1990, 29, 2332.

(b) Kawabata, J.; Mizutani, J. Phytochemistry 1992, 31, 1293.

(c) Kawabata, J.; Fukushi, E.; Mizutani, J. Phytochemistry 1995, 39, 121.

(d) Kawabata, J.; Funkushi, E.; Mizutani, J. Phytochemistry 1998, 47, 231.

(e) Wang, X. C.; Zhang, Y. N.; Wang, L. L.; Ma, S. P.; Liu, J. H.; Hu, L. H. J. Nat. Prod. 2008, 71, 674. 
[16] (a) Kawabata, J.; Fukushi, E.; Mizutani, J. Phytochemistry 1993, $32,1347$.

(b) Xiao, Z. Y.; Wang, X. C.; Zhang, G. P.; Huang, Z. L.; Hu, L. H. Helv. Chim. Acta 2010, 93, 803.

[17] (a) Yang, S. P.; Yue, J. M. Tetrahedron Lett. 2006, 47, 1129.

(b) Xu, Y. J.; Tang, C. P.; Ke, C. Q.; Zhang, J. B.; Weiss, H. C.; Gesing, E. R.; Ye, Y. J. Nat. Prod. 2007, 70, 1987.

[18] Wu, B.; Chen, J.; Qu, H. B.; Cheng, Y. Y. J. Nat. Prod. 2008, 71, 877.

[19] Ran, X. H.; Teng, F.; Chen, C. X.; Wei, G.; Hao, X. J.; Liu, H. Y. J. Nat. Prod. 2010, 73, 972.

[20] (a) Kim, S.-Y.; Kashiwada, Y.; Kawazoe, K.; Murakami, K.; Sun, H.-D.; Li, S.-L.; Takaishi, Y. Phytochem. Lett. 2009, 2, 110.

(b) Kim, S. Y.; Kashiwada, Y.; Kawazoe, K.; Murakami, K.; Sun, H. D.; Li, S. L.; Takaishi, Y. Tetrahedron Lett. 2009, 50, 6032.

(c) Kim, S.-Y.; Kashiwada, Y.; Kawazoe, K.; Murakami, K.; Sun, H.-D.; Li, S.-L.; Takaishi, Y. Chem. Pharm. Bull. 2011, 10, 1281.

[21] He, X. F.; Yin, S.; Ji, Y. C.; Su, Z. S.; Geng, M. Y.; Yue, J. M. J. Nat. Prod. 2010, 73, 45.

[22] Li, C. J.; Zhang, D. M.; Luo, Y. M.; Yu, S. S.; Li, Y.; Lu, Y. Phytochemistry 2008, 69, 2867.

[23] Fang, P.-L.; Cao, Y.-L.; Yan, H.; Pan, L.-L.; Liu, S.-C.; Gong, N.-B.; Lu, Y.; Chen, C.-X.; Zhong, H.-M.; Guo, Y.; Liu, H.-Y. J. Nat. Prod. 2011, 74, 1408

[24] He, X.-F.; Zhang, S.; Zhu, R.-X.; Yang, S.-P.; Yuan, T.; Yue, J.-M.
Tetrahedron 2011, 67, 3170 .

[25] Acebey, L.; Sauvain, M.; Beck, S.; Moulis, C.; Gimenez, A.; Jullian, V. Org. Lett. 2007, 9, 4693.

[26] Kwon, S. W.; Kim, Y. K.; Ryu, H. S.; Lee, H. K.; Kang, J. S.; Kim, H. M.; Hong, J. T.; Kim, Y.; Han, S.-B. Biomol. Ther. 2011, 19, 59.

[27] Kwon, O. E.; Lee, H. S.; Lee, S. W.; Bae, K. H.; Kim, K.; Hayashi, M.; Rho, M. C.; Kim, Y. K. J. Ethnopharmacol. 2006, 104, 270.

[28] Liu H.-Y.; Li, L.; Chen, C.-X.; Sun, L.; Teng, F.; Huang N,-X.; Ran, X.-H.; Ni, W.; Hao, X.-J.; Gao, S. CN 101671346, 2010 [Chem. Abstr. 2010, 152, 462967].

[29] Fenolon, T. W.; Schwaebisch, D.; Mayweg, A. V. W.; Lee, V.; Adlington, R. M.; Baldwin, J. E. Synlett 2007, 2679.

[30] Gordon, H. L.; Freeman, S.; Hudlicky, T. Synlett 2005, 2911.

[31] Liu, Y.; Nan, F. J. Tetrahedron Lett. 2010, 51, 1374.

[32] Qian, S.; Zhao, G. Synlett 2011, 722.

[33] Qian, S.; Zhao, G. Chem. Commun. 2012, 48, 3530.

[34] Lu, Y.-S.; Peng, X.-S. Org. Lett. 2011, 13, 2940.

[35] (a) Yue, G.; Yang, L.; Yuan, C.; Jiang, X.; Liu, B. Org. Lett. 2011, 13, 5406.

(b) Yue, G.; Yang, L.; Yuan, C.; Du, B.; Liu, B. Tetrahedron 2012, 68, 9624.

[36] Yue, G. Ph.D. Dissertation, Sichuan University, Chengdu, 2011 (in Chinese).

(乐贵洲，博士论文，四川大学，成都, 2011.) 